\title{
PEYORATIVOS DE GRUPO Y DISCURSO DE ODIO*
}

\section{GROUP PEJORATIVES AND HATE SPEECH}

Nicolás Lo Guercio https://orcid.org/0000-0003-4663-9080 nicolasloguercio@gmail.com Agencia Nacional de Promoción Cientifica y Tecnológica, Argentina

\begin{abstract}
The article advances a multidimensional semantics for slurs, according to which they express a standard at-issue content along with a conventional implicature. By contrast with existent conventional implicature accounts (McCready, 2010), it is argued that the not at-issue content of slurs is a property, instead of a proposition. When complemented with a dynamic pragmatic framework (Portner, 2004), the view implies that an utterance containing a slur carries two different discursive functions, that is, it updates two different components of context: the at-issue content updates the common ground; the not at-issue content updates the To-Do List. It is then showed how the view explains the way in which uses of slurs are capable of modifying permissibility facts and hence of bringing harm to their targets. Finally, the article shows how the present account deals with different conversational moves that slurs make available like those of propaganda, attack, complicity or appropriation.
\end{abstract}

Keywords Slurs. Hate Speech. Semantics. Pragmatics.

* Article submitted on 28/07/2020. Accepted on 26/11/2020. 
RESUMEN El artículo presenta una semántica multidimensional para los peyorativos de grupo de acuerdo con la cual estos codifican un contenido descriptivo y un contenido peyorativo implicaturado convencionalmente. A diferencia de otras propuestas en la misma linea (e.g. McCready, 2010), se argumenta que el contenido peyorativo es una propiedad, y no una proposición. A partir de un marco pragmático dinámico (Portner, 2004), se argumenta que el uso de un peyorativo de grupo instancia dos fuerzas discursivas, esto es, actualiza dos componentes del contexto conversacional. El contenido descriptivo actualiza el trasfondo común (Common Ground). El contenido not at-issue actualiza la lista de tareas pendientes (To-Do-List). Se muestra luego cómo la teoría explica el modo en que los peyorativos de grupo modifican hechos acerca de lo que es permisible en la conversación $y$, de este modo, causan y constituyen un daño para los grupos discriminados. Finalmente, se argumenta que la teoría permite dar cuenta de una variedad de movidas conversacionales habilitadas por estas expresiones, como la propaganda, el ataque, la complicidad o la apropiación.

Palabras clave Peyorativos de grupo. Lenguaje de odio. Semántica. Pragmática.

\section{Introducción}

Las lenguas naturales vehiculizan a menudo actos moralmente reprochables, esto es, permiten discriminar, subordinar o insultar a individuos y grupos. En este artículo discutiré un conjunto de expresiones especialmente apto para estos propósitos, los peyorativos de grupo (PG de aquí en más). Estos términos son utilizados para discriminar o expresar desprecio por ciertos grupos, típicamente identificados en virtud de su preferencia sexual, etnia, nacionalidad, género, clase, orientación política o religión, entre otros.

Los PG poseen características semántico-pragmáticas particulares. En primer lugar, expresan dos tipos de contenido. A modo de ilustración, considere las siguientes oraciones:

1. Juan es bolita. ${ }^{1}$

2. Juan es boliviano.

1 El término 'bolita' es un PG utilizado en el español del Río de la Plata para referir a las personas nacidas en Bolivia. Quisiera dejar en claro que todos los PG que aparecen en este artículo están mencionados (no usados) con fines ilustrativos, y que condeno todas las actitudes y/o estereotipos comúnmente asociados a estas expresiones. 
Intuitivamente, (1) comunica el mismo contenido que (2), esto es, Juan es boliviano. Sin embargo, la primera indica, en virtud de la ocurrencia del PG 'bolita', que el hablante tiene una actitud negativa hacia los bolivianos, mientras que la segunda se limita a señalar la procedencia del sujeto de la oración. Denominaré a estos dos tipos de contenido contenido descriptivo y contenido peyorativo.

En segundo lugar, los PG se comportan de un modo particular en ciertos entornos lingüísticos. Por ejemplo, el contenido peyorativo, a diferencia del contenido descriptivo, es difícil de negar de manera directa, esto es, sin alterar el flujo natural de la conversación: ${ }^{2}$

3.

a. Juan es bolita.

b. No es cierto, Juan no es boliviano.

c. \#No es cierto, los bolivianos no son despreciables.

Por otra parte, la negación explícita no parece afectar al contenido peyorativo, esto es, una oración como (4) resulta igual de ofensiva que (3a):

4. Juan no es bolita.

Este tipo de interacción con operadores veritativo-condicionales sugiere que el contenido peyorativo no forma parte de las condiciones de verdad de la oración. ¿Cómo se comunican entonces estos contenidos? El consenso en la bibliografía sobre la cuestión es que el contenido descriptivo funciona en el nivel de las condiciones de verdad de la oración. Sin embargo, existe un intenso debate con respecto al contenido peyorativo. Muchas propuestas buscan semantizarlo de uno u otro modo. Hom (2008) y Hom y May (2013) sitúan el contenido peyorativo en las condiciones de verdad de la oración, Schlenker (2007) y Cepollaro (2017) lo tratan como una presuposición, y Orlando y Saab (2020), McCready (2010), Predelli (2013) y Gutzmann (2015) desarrollan diferentes variantes de una teoría de implicaturas convencionales. Otros enfoques, en cambio, caracterizan el fenómeno en términos pragmáticos. Anderson y Lepore (2013) afirman que los PG funcionan como palabras tabúes.

2 Además, la interpretación del significado expresivo no es fácilmente desplazable de la situación de emisión (el hablante, el tiempo, el mundo de la emisión, etc.), incluso cuando la expresión es usada bajo el alcance de operadores de actitud proposicional, modales, temporales, reportes indirectos, condicionales, etc. Otras características (ausentes en el significado descriptivo) son la dificultad para parafrasear su significado en términos proposicionales (inefabilidad descriptiva), la posibilidad de repetir expresiones con significado expresivo sin redundancia (repetibilidad) o la dificultad para suspender sus efectos contextuales, incluso cuando solo se los mencione (inmediatez). 
Bolinger (2017) sostiene que el contenido peyorativo resulta del contraste léxico entre expresiones regularmente asociadas con ciertas actitudes y creencias, mientras que Nunberg (2018) lo deriva como una implicatura conversacional basada en la violación de la Máxima de Modo.

En tercer lugar, los PG resultan filosóficamente interesantes por su dimensión socio-política. Como resaltan Langton, Haslanger y Anderson (2012), estas expresiones contribuyen al discurso de odio, en tanto sus usos comunican un mensaje de inferioridad dirigido a un grupo históricamente oprimido y son persecutorios, están cargados de odio y resultan degradantes (Matsuda, 2018, p. 36). Este fenómeno tiene varias particularidades (Langton, 1993; McGowan, 2004, 2019; Langton y West, 1999; Maitra, 2012; Tirrell, 2012, entre muchos otros). Por un lado, el discurso de odio causa daño. Matsuda (2018) señala que las víctimas pueden experimentar síntomas psicológicos y emocionales que incluyen miedo, aceleración del pulso cardíaco, estrés postraumático, hipertensión, etc., además de efectos profundos en la autoestima. Las víctimas ven afectada, además, su libertad personal:

\footnotetext{
para evitar recibir mensajes de odio, las víctimas deben renunciar a sus trabajos, abandonar su educación, dejar sus hogares, evitar ciertos lugares públicos, restringir el ejercicio de su derecho a la libre expresión y modificar de otros modos su conducta o comportamiento (Matsuda, 2018, p. 24) [Traducción propia].
}

Sin embargo, no todo discurso de odio daña del mismo modo. Conviene distinguir entre discursos que causan daño y discursos que constituyen un daño (MacKinnon, 1987). Esta distinción suele elucidarse a partir de las categorías austinianas de acto locucionario, ilocucionario y perlocucionario (Langton, 1993). A grandes rasgos, un acto locucionario consiste en la realización de una emisión significativa en virtud de sus aspectos sintácticos, semánticos y fonológicos, un acto ilocucionario es aquel realizado a través de una emisión con cierta 'fuerza' (afirmación, promesa, invitación, orden, etc.), y un acto perlocucionario engloba los efectos causales de la emisión que resultan de su funcionamiento lingüístico. Un discurso causa daño cuando este puede ser caracterizado como un efecto perlocucionario de la emisión. Un discurso constituye un daño, en cambio, cuando promueve, instituye o legitima, en virtud de su fuerza ilocucionaria, normas sociales que prescriben un daño material, moral, epistémico, etc., hacia ciertos grupos. Langton $(1993,2015)$ argumenta que algunas emisiones requieren, en virtud de las condiciones de felicidad asociadas a su fuerza ilocucionaria, que ciertos grupos ocupen un lugar subordinado/dominante, determinados roles sociales, etc. Por ejemplo, en el contexto del Apartheid, un cartel en el frente de un negocio que reza 'whites only' (solo blancos) tiene como condición de felicidad la vigencia de 
cierta jerarquía donde los blancos ocupan un lugar dominante y los individuos de otras etnias un lugar subordinado. Esta emisión causa un daño - tiene efectos perlocucionarios dañinos - y, además, en caso de ser exitosa, instituye o contribuye a mantener en vigencia, en virtud del acto de habla realizado, una norma social que jerarquiza a los individuos por su color de piel y prescribe así un daño material y moral hacia las personas de otras etnias. En este caso, el discurso constituye un daño.

Por otro lado, lo dicho pone en relieve la pregunta por la fuerza ilocucionaria de diferentes instancias de discurso de odio asociadas a PG. Langton, Haslanger y Anderson (2012, pp. 758-759) identifican tres fuerzas ilocucionarias relacionadas con el discurso de odio: (i) propaganda, (ii) ataque y (iii) discurso de autoridad. La propaganda es un tipo de discurso que promueve el odio y la violencia. Los usos como ataque consisten en una mera expresión de odio. Por último, a través del discurso de autoridad los hablantes instituyen ciertas normas sociales en virtud de su rol de autoridad. El punto de estos últimos es privar a ciertos grupos de algunos de sus derechos. ${ }^{3}$ Además de estos usos, discutiré en este artículo lo que Davis y McCready (2017) denominan usos cómplices, en los cuales el PG es utilizado entre individuos que comparten el prejuicio en cuestión.

Un último fenómeno asociado a los PG es el de la apropiación (Bianchi, 2014; Brontsema, 2004). Esta consiste en la adopción del término en cuestión por el grupo destinatario, invirtiendo su sesgo peyorativo, tal vez con el fin de comunicar un sentimiento de solidaridad o camaradería. Un ejemplo puede ser la apropiación por parte de grupos afroamericanos de la expresión 'Nigger' en los Estados Unidos. Una explicación adecuada de estos términos debería arrojar luz sobre la cuestión.

He presentado hasta aquí cuatro desafíos que una teoría sobre los PG debe afrontar. ${ }^{4}$ En primer lugar, esta debe precisar el mecanismo a través del cual comunican un contenido peyorativo. En segundo lugar, debe proporcionar una explicación general del modo en que modifican hechos acerca de lo que es permisible. En este punto, los PG introducen una dificultad adicional con

3 Como su nombre lo indica, el discurso de autoridad consiste en un tipo de discurso de odio realizado desde una posición de autoridad, e.g. un legislador blanco pretoriano que decreta, en el contexto apropiado, 'los individuos negros no tienen permitido votar' durante el apartheid (Langton, 1993, p. 302). Véase también Maitra (2012). No discutiré estos usos en detalle en este artículo.

4 La lista no es exhaustiva. Otras cuestiones a explicar, que no trataré en este artículo, son la diferencia de intensidad entre diferentes PG, la diferencia entre distintos peyorativos para el mismo grupo y la posibilidad de usos no peyorativos y no apropiados en algunos contextos particulares. Además, asumiré que una teoría de implicaturas convencionales como la que defenderé en la sección 3 permite lidiar relativamente bien con varios de los fenómenos de no-desplazabilidad, repetibilidad e inefabilidad mencionados en la nota 7 (cf. McCready, 2010; Gutzmann, 2015). 
respecto a otros casos de discurso de odio, pues parece que la explicación no puede en principio ser completamente pragmática (Langton, 2015), sino que involucra una interacción compleja entre el significado convencional de los términos y mecanismos pragmáticos. ${ }^{5}$ En tercer lugar, la teoría debe dar cuenta del perfil ilocucionario de los PG y el modo en que estos constituyen un daño. Finalmente, la explicación debe arrojar luz sobre el fenómeno de la apropiación.

El artículo está estructurado como sigue: en la próxima sección presentaré el marco teórico necesario para desarrollar mi propuesta sobre la semántica y la pragmática de los PG. En la sección 3 argumentaré que los PG expresan una implicatura convencional, en la línea de McCready (2010) y Gutzmann (2015). Sin embargo, defenderé que el contenido implicado convencionalmente es una propiedad, a diferencia de estas propuestas. En la sección 4 mostraré cómo la teoría semántica propuesta más la adopción de un marco de pragmática dinámica permiten dar cuenta de las características del discurso de odio antes discutidas. Finalmente, discutiré una propuesta alternativa en la misma línea que la aquí presentada (Poppa-Wyatt y Wyatt, 2017), y mostraré algunos inconvenientes relacionados con la misma.

\section{Marco teórico}

\subsection{Pragmática dinámica}

Asumiré en este trabajo un marco de pragmática dinámica en la línea de Stalnaker (1999) y Portner (2004). Asimismo, asumiré que la fuerza ilocucionaria puede derivarse a partir del contenido semántico, de la fuerza discursiva y del contexto de emisión (véase Roberts, 2018). La idea general es la siguiente. Los tipos oracionales constituyen un sistema universal y cerrado definido gramaticalmente. Existen tres tipos oracionales universales: declarativo, interrogativo e imperativo. Cada uno está asociado con una fuerza discursiva diferente (Portner, 2004; Roberts, 2018). Asumiré que cada tipo oracional determina un objeto semántico tradicional: las oraciones declarativas expresan una proposición, las interrogativas una pregunta, i.e. un conjunto de proposiciones, y las imperativas una propiedad. Los tipos oracionales y los contenidos semánticos correspondientes están conectados por defecto con

5 Esta restricción deriva de mi propia posición acerca de la semántica de los PG, a favor de la cual argumentaré en la próxima sección. Como se dijo antes, existen teorías puramente pragmáticas de los PG (Bolinger, 2017; Nunberg, 2018). Por razones de espacio no puedo discutirlas en detalle. Véase Cepollaro (2017) para críticas a estas posiciones. 
fuerzas discursivas específicas, esto es, la fuerza discursiva está determinada solo de manera indirecta, sobre la base de estos significados (Portner, 2004, p. 236). ${ }^{6}$

Las diferentes fuerzas discursivas son entendidas como modos particulares de actualizar algún componente del contexto conversacional, donde cada una es una instancia de un esquema universal de actualización contextual del tipo "tome un conjunto de Xs y una X, y agregue la nueva X al conjunto" (Portner, 2004, p. 238) [Traducción propia]. Las oraciones declarativas tienen por defecto fuerza asertiva. Una aserción es una propuesta de actualización del trasfondo común (Common Ground, CG), esto es, el conjunto de proposiciones comúnmente aceptado por los participantes de la conversación. Una pregunta es una propuesta para actualizar el conjunto de preguntas bajo discusión (Question Under Discussion, QUD), esto es, el conjunto de cuestiones que los hablantes acuerdan que deben ser resueltas en la conversación. Finalmente, un pedido (entendido en este contexto como una función discursiva general) es una propuesta para actualizar la lista de tareas (To-Do List, TDL), esto es, un conjunto de propiedades que cierto individuo se ha comprometido públicamente a volver verdaderas de sí mismo (las TDLs están indexadas a participantes específicos de la conversación, cada participante tiene su propia TDL). Las oraciones declarativas tienen la función discursiva general de la aserción, las interrogativas la de la pregunta y las imperativas la del pedido. ${ }^{7}$ La correlación entre tipos oracionales, tipos semánticos y fuerza discursiva es regular y se da por defecto, pero es, sin embargo, revocable. Por ejemplo: la oración " ¡Te vas a sentar!" es declarativa y expresa una proposición, pero puede ser usada, en el contexto apropiado, para actualizar la TDL en lugar del CG (e.g., para dar una orden).

Finalmente, adoptaré la posición de Roberts (2018, pp. 337-338), de acuerdo con la cual el acto de habla realizado por una emisión puede ser predicho en virtud de la semántica de los constituyentes emitidos, el contexto de emisión, inferencias de tipo griceano y principios generales de interpretación del discurso (relevancia, cooperatividad, racionalidad, etc.). De este modo, asumiré en lo que sigue que la conexión entre fuerza discursiva y tipo de acto de habla es pragmática, no semántica.

6 Asumo aquí lo que Portner (2018, pp. 153-157) denomina enfoque composicional.

7 Se entiende aquí 'pedido' como una fuerza discursiva general que engloba diferentes actos de habla directivos como órdenes, consejos, invitaciones, etc. 


\subsection{Imperativos}

La propuesta que defenderé en la siguiente sección aprovecha cierta analogía entre PG e imperativos. Por ese motivo, en esta subsección discutiré algunas características de los imperativos que serán relevantes para la discusión posterior. De acuerdo con el enfoque dinámico introducido en la subsección anterior, las oraciones imperativas expresan una propiedad, esto es, un contenido con el tipo semántico $<$ e, $\mathrm{t}>$, y su fuerza discursiva canónica es la de actualizar la TDL indexada al destinatario. Más formalmente, los imperativos actualizan el contexto como sigue:

5. $C+\phi_{\text {imp }}=\left\langle C G, Q S, T\left[\frac{\text { destinatario }}{\left(T(\text { destinatario }) \cup\left\{\llbracket \phi_{\text {imp }} \rrbracket\right\}\right)}\right]\right\rangle$

(Portner, 2007, p. 357)

Usualmente, los imperativos portan fuerza directiva. La fuerza directiva se caracteriza típicamente como un intento de generar en el destinatario la intención de actuar de cierto modo. Así, es intuitivo interpretar a las propiedades presentes en la TDL como acciones que el destinatario se ha comprometido a llevar a cabo. Sin embargo, una emisión con fuerza discursiva directiva puede funcionar como una interpelación al destinatario para que adopte determinadas actitudes no-cognitivas (e.g. enojo), o disposiciones emocionales, por ejemplo: "¡Calmáte!”, “¡No te enojes!”, etc. Así, como nota Portner, la TDL contiene propiedades que el destinatario se ha comprometido a volver verdaderas de sí mismo, sin que esté necesariamente especificado qué acciones debe adoptar para lograr ese objetivo. ${ }^{8}$ Formalmente, la semántica para un imperativo es la siguiente:

6. ¡Andáte!

7. $\llbracket$ Andáte $\rrbracket^{\mathrm{w}^{*}, \mathrm{c}}=\lambda w \cdot \lambda x: x=\operatorname{destinatario}(c) . x$ se va en $w^{9}$

En cuanto a la pragmática, Portner argumenta que el CG y la TDL están íntimamente relacionados en la interpretación de los imperativos. El primero cumple un rol similar al de la base modal en la semántica de Kratzer (2012,

8 Véase Portner (2007, nota 2).

9 Aquí la condición de que la propiedad sea verdadera con respecto al destinatario está incluida como una presuposición. El imperativo denota, de este modo, una función parcial que devuelve un resultado indeterminado si el argumento no es el destinatario. 
cap. 1-2), es decir, determina el conjunto de mundos posibles contextualmente relevantes para interpretar el imperativo. La segunda cumple un rol análogo al de la fuente de ordenación (ordering source) en la semántica de Kratzer: la TDL ordena los mundos del CG de acuerdo a cuántas propiedades de la TDL son verdaderas en esos mundos, situando más arriba en el orden aquellos donde se satisfacen más propiedades.

Este enfoque tiene dos ventajas. Primero, permite explicar las diferentes fuerzas ilocucionarias asociadas con los imperativos como ordenar, sugerir, aconsejar, pedir, advertir, etc. Según esta posición, hay varias TDL asociadas con cada participante de la conversación, correspondientes a los diferentes tipos de fuentes de ordenación en la semántica de Kratzer para modales: deóntica, bulética, teleológica, etc. Todos los actos de habla mencionados emergen a partir de la misma fuerza discursiva, esto es, la de agregar una propiedad a la TDL, pero difieren en cuanto a la naturaleza de la TDL involucrada y, plausiblemente, con respecto a otros factores contextuales y las intenciones comunicativas involucradas. Por ejemplo, una orden, además de requerir, como una condición de felicidad, autoridad por parte del hablante, agrega una propiedad a la TDL deóntica asociada con el destinatario. Una vez aceptada, el destinatario asume públicamente una nueva obligación, a saber, la de hacer verdadera la propiedad en su TDL. Luego, los mundos de CG son ordenados de acuerdo con el número de propiedades de su TDL deóntica que son satisfechas en esos mundos.

Segundo, y relacionado con lo anterior, la posición explica la íntima relación entre los imperativos y los modales. El uso de imperativos licencia conversacionalmente la emisión de un modal correspondiente, y viceversa:

8. ¡Sentáte! [dicho a Juan]

9. Juan debería sentarse [modal deóntico].

10. ¡Cométe una galletita! [dicho a Mariana]

11. Mariana debería comerse una galletita [modal bulético].

La teoría de Portner explica esta regularidad como sigue. Supongamos que doy una orden y esta es aceptada por mi interlocutor. Este adquiere así una nueva obligación, que es capturada por una proposición modal que establece un deber de algún tipo. A su vez, el hecho de haber asumido este compromiso es un hecho manifiesto en el contexto y, por lo tanto, es consignado en el CG, que se actualiza con la proposición modal correspondiente. Algo análogo ocurre con las invitaciones, sugerencias, consejos, etc., y las modalidades correspondientes (bulética, teleológica, etc.). 


\section{Una teoría bidimensional para los peyorativos de grupo}

Como fue indicado anteriormente, existen varias propuestas para dar cuenta del modo en que los PG comunican contenido peyorativo. En esta sección desarrollaré una versión propia de la teoría de implicaturas convencionales y mostraré cómo este enfoque, junto con una teoría pragmática dinámica como la presentada en la sección anterior, permite dar cuenta de los desafíos señalados en la introducción.

\subsection{Por qué una teoría de implicaturas convencionales}

La teoría de implicaturas convencionales (McCready, 2010; Gutzmann, 2015) está motivada por el patrón de proyección de los PG, así como por algunas consideraciones conceptuales. Por un lado, el contenido peyorativo proyecta por fuera de los operadores veritativo-condicionales típicos, algo que resulta problemático para las teorías de condiciones de verdad (Hom, 2008; Hom y May, 2013): ${ }^{10}$

\section{Juan es bolita.}

13. Juan no es bolita.

14. Si invitás a un bolita a la fiesta, yo no voy a ir.

15.¿Cuántos bolitas hay en tu equipo?

16. No estoy seguro, pero Juan podría ser bolita.

Las oraciones (12)-(16) resultan todas ofensivas, aun cuando 'bolita' esté bajo un operador veritativo-condicional o dentro de una oración interrogativa. Esto sugiere que su contenido peyorativo no forma parte de las condiciones de verdad. Una respuesta intuitiva ante la naturaleza proyectiva del contenido peyorativo es adoptar un enfoque presuposicional. Sin embargo, el patrón de proyección del contenido peyorativo parece diferir del de las presuposiciones:

17. María cree que Juan es bolita.

18. María cree que Juan ganó un partido de fútbol.

19. Si los bolivianos son despreciables, Juan es bolita.

20. Si Juan tiene un hermano, su hermano es profesor.

10 Hom (2008) y Hom y May (2013) están al tanto del problema. Los autores intentan dar cuenta de la cuestión (i) distinguiendo entre ofensa y derogación y (ii) relativizando el problema en virtud de una serie de presuntos contraejemplos donde se hace un uso no-apropiado y no-proyectivo. Véase Sennet y Copp (2015) y Cepollaro y Thommen (2019) para una discusión crítica de sus propuestas. 
Mientras que el contenido peyorativo de (17) proyecta por fuera de la subordinada, la presuposición 'Juan jugó un partido de fútbol' es bloqueada por el verbo de creencia en (18). Del mismo modo, la presuposición en (20) es ligada por el antecedente del condicional, mientras que esto no ocurre con el contenido peyorativo en (19). Así, el patrón de proyección de los PG parece asemejarse más al de los expresivos, que portan implicaturas convencionales (Potts, 2005), que al de otras expresiones con contenido presuposicional:

21. María cree que el puto profesor la va a reprobar (el hablante tiene una actitud negativa hacia el profesor).

22. Si tuviera una opinión negativa sobre el profesor, el puto profesor me reprobaría (el hablante tiene una actitud negativa hacia el profesor).

Existen también razones conceptuales que favorecen, en principio, a las teorías de implicaturas convencionales. Como se dijo en la sección 2, las aserciones, cuando son aceptadas, actualizan el CG, esto es, actualizan el conjunto de creencias comunes entre el hablante y el oyente con la proposición afirmada. Las presuposiciones, por su parte, imponen una restricción a esta actualización: la proposición expresada por una oración que presupone el contenido $p$ pasa a formar parte del CG solo en la medida en que $p$ forme también parte del CG. Considere, por ejemplo, la oración 'Llevé a mi perro al veterinario'. La proposición afirmada, que el hablante llevó a su perro al veterinario, solo actualiza el CG en la medida en que su presuposición, que el hablante tiene un perro, es también parte del CG. Si esto no ocurre, en cambio, la aserción puede ser objetada: ‘¿Espera un minuto! ¡No sabía que tenías un perro!' En otras palabras, aceptar que el hablante llevó a su perro al veterinario parece requerir una aceptación previa, a saber, que el hablante tiene un perro. ${ }^{11}$

No parece ocurrir lo mismo en el caso de los PG. Considérese de nuevo (1) (repetida aquí como (23)):

\section{Juan es bolita.}

En un análisis presuposicional, el contenido descriptivo afirmado, que Juan es boliviano, solo puede actualizar el CG en la medida en que su contenido peyorativo, que el hablante tiene una actitud negativa hacia los bolivianos,

11 Estoy aquí simplificando la cuestión pues, dependiendo del caso, el hablante puede acomodar la presuposición en cuestión (cf. von Fintel 2008), esto es, la aceptación de la presuposición puede ser posterior a la realización de la emisión (aunque no a la actualización del CG). 
forme también parte del CG. Pero esto no parece ser el caso: no es necesario aceptar el contenido peyorativo, una proposición sobre las actitudes del hablante, o sobre el estatus moral de los bolivianos, para agregar al CG el contenido descriptivo, i.e. la proposición Juan es boliviano.

Aunque la cuestión requiere una discusión más detallada, lo dicho sirve al menos para motivar la búsqueda de una teoría alternativa, en particular una teoría de implicaturas convencionales. ${ }^{12}$ Ahora bien, para llevar adelante esta tarea es necesario proporcionar una semántica explícita para los PG. En la próxima subsección desarrollaré una propuesta en esta dirección.

\subsection{Una semántica para los $P G$}

Una de las teorías de implicaturas convencionales sobre PG más desarrollada es la de McCready (2010), inspirada en Potts (2005). ${ }^{13}$ Según este enfoque, un PG como 'bolita' expresa dos dimensiones de contenido: en el nivel veritativo-condicional, también denominado contenido at-issue, denota la propiedad de ser boliviano; en el nivel implicaturado convencionalmente, también denominado not at-issue, expresa una proposición como Los bolivianos son malos (donde 'malo' indica vagamente una actitud negativa del hablante):

24. $\llbracket$ Boliviano $\rrbracket=\lambda \mathrm{x}$ : Boliviano( $\mathrm{x}):<\mathrm{e}, \mathrm{t}>^{a} \diamond \operatorname{Malo}\left({ }^{\prime}\right.$ Boliviano $): \mathrm{t}^{c}$

Adoptaré aquí la semántica multidimensional de tipos desarrollada por McCready. En este sistema existen dos tipos de contenido not at-issue: el contenido not at-issue proposicional constituye una implicatura convencional; el contenido not at-issue no proposicional constituye un contenido shunting. En este trabajo defenderé, sin embargo, contra McCready, que el contenido not atissue de los PG tiene la forma de una propiedad (en lugar de una proposición). Argumentaré que esta modificación permite dar cuenta del modo en que los PG modifican hechos acerca de lo que es permisible y arroja luz sobre su perfil ilocucionario y el modo en que este se relaciona con su significado literal.

De acuerdo con la posición aquí defendida un PG como 'bolita' tiene una dimensión at-issue que denota la propiedad 'boliviano' y una dimensión not at-issue que expresa una propiedad diferente, la de despreciar a los bolivianos:

12 Véase Saab (2020) y Lo Guercio (2021) para otros argumentos contrarios a las teorías presuposicionales.

13 Véase también Gutzmann (2015) y Predelli (2013). Hom (2008) y Hom and May (2013) desarrollan críticas a las teorias de implicaturas convencionales. Kratzer (1999) y Anand (2007) presentan algunos ejemplos problemáticos. 
25. $\llbracket$ Boliviano $\rrbracket=\lambda x$ : Boliviano $(x) \downarrow \lambda x$ : despreciar $\left({ }^{(}\right.$Boliviano $)(x):<\mathrm{e}, \mathrm{t}^{\mathrm{a}} \mathrm{x}<\mathrm{e}^{\mathrm{s}}$, $t^{\mathrm{s}>}$

Esta modificación requiere realizar algunos ajustes al sistema de McCready. En primer lugar, es necesario introducir un nuevo tipo semántico. Llamemos al nuevo sistema $L_{C I}{ }^{++}$(basado en el sistema de McCready $L_{C I}{ }^{+}$). El sistema de tipos de $L_{C I}{ }^{++}$es idéntico al de $L_{C I}{ }^{+}$(véase McCready, 2010, apéndice) con el siguiente agregado:

26. Si $\sigma$ y $\tau$ son tipos at-issue de $L_{C I}^{++}$y $\zeta$ y $v$ son tipos shunting de $L_{C I}^{++}$ entonces $<\sigma, \tau>x<\zeta$, $v>$ es un tipo mixto de $L_{C I}^{++}$

En segundo lugar, introduciré nuevas reglas para interpretar este tipo semántico, pues las reglas de $\mathrm{L}_{\mathrm{CI}}^{+}$no pueden hacerlo. Para ver el punto, considérese las reglas relevantes en el sistema de McCready (2010, p. 20):

27.

$$
\frac{\alpha \diamond \beta:<\sigma^{a}, \tau^{a}>x<\sigma^{a}, v^{s}>, \gamma: \sigma^{a}}{\alpha(\gamma) \diamond \beta(\gamma): \tau^{a} \times v^{s}}
$$

28.

$$
\alpha \diamond \beta: \sigma^{a} \times t^{s}
$$

$$
\alpha: \sigma^{a} \bullet \beta: \mathfrak{t}^{s}
$$

La primera regla interpreta el caso en el que el argumento para el contenido at-issue y el contenido not at-issue es del mismo tipo, esto es, ambos toman un argumento at-issue. La segunda estipula que cuando la derivación llega a algo de tipo proposicional en el nivel shunting, pasa al nivel de implicatura convencional. Así, tenemos en el sistema de McCready expresiones que codifican una propiedad en ambas dimensiones de significado, pero ambas propiedades toman un argumento at-issue. Estas reglas no permiten interpretar el nuevo tipo introducido más arriba, cuya dimensión not at-issue toma a su 
vez un argumento not at-issue. En un intento por preservar el sistema de McCready, podríamos estipular que la dimensión not at-issue de los PG tomen un argumento at-issue. Esto arrojaría, sin embargo, resultados inaceptables. Para ver el punto, considérese la derivación de la oración (23):

Juan: $e^{a} \quad \lambda x$ : Boliviano $(x) \diamond \lambda x$ : despreciar( $\left({ }^{\cap}\right.$ Boliviano $)(x):<e, t^{a} \times<e^{a}, t^{s}>$ Boliviano(Juan) despreciar $\left({ }^{\cap}\right.$ Boliviano)(Juan): $t^{a} \times t^{s}$

Boliviano(Juan): $\mathrm{t}^{a} \bullet \operatorname{despreciar}\left({ }^{\cap}\right.$ Boliviano)(Juan): $t^{c}$

Esta derivación arroja un resultado indeseable en el nivel not at-issue, esto es, la proposición Juan desprecia a los bolivianos. Para solucionar el problema necesitamos una regla que permita un tipo mixto en el que el argumento para la dimensión shunting sea también de tipo shunting:

29.

$$
\frac{\alpha \diamond \beta:<\sigma, \tau>^{a} \times<\zeta^{s}, v^{s}>, \gamma: \sigma^{\mathrm{a}}}{\alpha(\gamma) \diamond \beta: \tau^{a} \times<\zeta^{s}, v^{s}>}
$$

Esta regla arroja los resultados deseados:

Juan: $e^{a} \quad \lambda x$ : Boliviano( $(x) \downarrow \lambda x$ : despreciar( ${ }^{\cap}$ Boliviano $)(x):<e,>^{a} \times<e^{s}, t^{s}>$

Boliviano(Juan) $\lambda x$ : despreciar( $\left({ }^{\cap}\right.$ Boliviano $)(x): t^{a} \times<e^{s}, t^{s}>$

De acuerdo con esta teoría, la propiedad de tipo shunting nunca alcanza estatus proposicional, de modo que nunca pasa a la dimensión implicaturada convencionalmente. Este ajuste soluciona el problema anterior, pero conduce a uno nuevo, relacionado con la regla de interpretación para árboles sintácticos completos. El problema es que, tal como está formulada en $L_{C I}{ }^{+}$, la regla compila solamente los contenidos not at-issue de tipo proposicional: 


\section{Interpretación generalizada $\left(L_{C I}^{+}\right)$}

Sea $\Pi$ una prueba para un árbol sintáctico con el término at-issue $\alpha: \sigma^{\mathrm{a}}$ en su nodo raíz, y los términos $\beta_{1}: \mathrm{t}^{\{\mathrm{c}, \mathrm{s}\}}, \ldots, \beta_{\mathrm{n}}: \mathrm{t}^{\{\mathrm{c}, \mathrm{s}\}}$ en sus nodos subordinados. La interpretación de $\Pi$ es $<\left[\left[\alpha: \sigma^{\mathrm{a}}\right]\right],\left\{\left[\left[\beta_{1}: \mathrm{t}^{\{\mathrm{c}, \mathrm{s}\}}\right]\right], \ldots,\left[\left[\beta_{\mathrm{n}}: \mathrm{t}^{\{\mathrm{c}, \mathrm{s}\}}\right]\right]\right\}>$.

Sea $\Pi$ una prueba para un árbol sintáctico con el término $a t$-issue $\alpha: \sigma^{\{c, s\}}$ en su nodo raíz y los términos $\beta_{1}: \mathrm{t}^{\{\mathrm{c}, \mathrm{s}\}}, \ldots, \beta_{\mathrm{n}}: \mathrm{t}^{\{\mathrm{c}, \mathrm{s}\}}$ en sus nodos subordinados. La interpretación de $\Pi$ es $<\mathrm{T},\left[\left[\alpha: \sigma^{\{c, s\}}\right]\right],\left\{\left[\left[\beta_{1}: \mathrm{t}^{\{\mathrm{c}, \mathrm{s}\}}\right]\right], \ldots,\left[\left[\beta_{\mathrm{n}}: \mathrm{t}^{\{\mathrm{c}, \mathrm{s}\}}\right]\right]\right\}>{ }^{14}$

(McCready, 2010, p. 32)

Necesitamos introducir una modificación más, esta vez en la regla de interpretación de árboles, para que tomen en cuenta propiedades shunting que sobrevivan como tales hasta el final de la derivación:

\section{Interpretación generalizada $\left(L_{C I}^{++}\right)$}

Sea $\Pi$ una prueba para un árbol sintáctico con el término at-issue $\alpha: \sigma^{\mathrm{a}}$ en su nodo raíz y los nodos subordinados $\beta_{1}: t^{\{\mathrm{c}, \mathrm{s}\}}, \ldots, \beta_{\mathrm{n}}: t^{\{\mathrm{c}, \mathrm{s}\}}$, y $\gamma_{1}:<e, t^{\{\mathrm{c}, \mathrm{s}\}}, \ldots, \gamma_{\mathrm{n}}:<e$, $t^{\{\mathrm{c}, \mathrm{s}\}}$. La interpretación de $\Pi$ es $<\left[\left[\alpha: \sigma^{\mathrm{a}}\right]\right],\left\{\left[\left[\beta_{1}: t^{\{\mathrm{c}, \mathrm{s}\}}\right]\right], \ldots,\left[\left[\beta_{\mathrm{n}}: t^{\{\mathrm{c}, \mathrm{s}\}}\right]\right]\right\},\left\{\left[\left[\gamma_{1}:<e\right.\right.\right.$, $\left.\left.\left.t^{\{c, s\}}\right]\right], \ldots,\left[\left[\gamma_{\mathrm{n}}:<e, t>^{\{c, s\}}\right]\right\}\right\}$.

Sea $\Pi$ una prueba para un árbol sintáctico con el término at-issue $\alpha: \sigma^{\{c, s\}}$ en su nodo raíz y los nodos subordinados $\beta_{1}: t^{\{\mathrm{c}, \mathrm{s}\}}, \ldots, \beta_{\mathrm{n}}: t^{\{\mathrm{c}, \mathrm{s}\}}$, y $\gamma_{1}:<e, t^{\{\mathrm{c}, \mathrm{s}\}}, \ldots, \gamma_{\mathrm{n}}:<e$, $t^{\{\mathrm{c}, \mathrm{s}\}}$. La interpretación de $\Pi$ es $<\mathrm{T},\left[\left[\alpha: \sigma^{\{\mathrm{c}, \mathrm{s}\}}\right]\right],\left\{\left[\left[\beta_{1}: t^{\{\mathrm{c}, \mathrm{s}\}}\right]\right], \ldots,\left[\left[\beta_{\mathrm{n}}: t^{\{\mathrm{c}, \mathrm{s}\}}\right]\right]\right\}$, $\left\{\left[\left[\gamma_{1}:<e, t^{\{s\}}\right]\right], \ldots,\left[\left[\gamma_{\mathrm{n}}:<e, t^{\{\mathrm{s}\}}\right]\right]\right\}>$.

Esta regla genera el resultado deseado: compila todos los contenidos at-issue, todos los contenidos proposicionales not at-issue y todos los contenidos noproposicionales not at-issue. 


\subsection{Una pragmática para los $P G$}

Ahora bien, la semántica propuesta va en paralelo con una dinámica conversacional. Como dije anteriormente, asumo aquí que los tipos semánticos generan por defecto una fuerza discursiva, entendida como cierto potencial de actualización de algún componente del contexto. Así, el uso de un PG como 'bolita' actualiza dos componentes diferentes del contexto, que se corresponden con las dos dimensiones de significado de la expresión, la descriptiva y la peyorativa. Una oración como (23) expresa, en el nivel del significado at-issue, la proposición Juan es boliviano. Por defecto, la fuerza discursiva es la de una aserción, de modo que (23) actualiza el CG con la proposición en cuestión. Sin embargo, según la semántica discutida en la subsección anterior, en el nivel not at-issue los PG expresan una propiedad, que como no se satura con ningún argumento, llega como tal al final de la derivación. Consecuentemente, (23) tiene, por defecto, la fuerza discursiva de un pedido y, en consecuencia, actualiza la TDL.

Recuerde, sin embargo, que cada participante de la conversación tiene asignada su propia TDL. Además, vimos que existen diferentes tipos de TDL (deóntica, bulética, teleológica). La pregunta es ¿cuál de estas TDL es actualizada por el uso del PG? En la presente propuesta, la cuestión queda determinada por el contexto. Para decirlo todavía en términos muy generales (analizaremos las diferentes fuerzas ilocucionarias asociadas a los PG más abajo), el uso de un PG actualiza alguna TDL (deóntica, bulética, teleológica) asignada a alguno de los participantes de la conversación (el hablante, el oyente o ambos) y, de este modo, los compromete públicamente a volver la propiedad relevante (despreciar al grupo en cuestión) verdadera de sí mismos. ${ }^{14}$

Ahora bien, como se dijo anteriormente, la TDL cumple una función análoga a la de la ordering source en la semántica kratzeriana para los modales, esto es, ordena los mundos del CG de acuerdo a la cantidad de propiedades que resultan verdaderas en dichos mundos. Así, el uso de un PG establece que los mundos donde se satisface la propiedad en cuestión, esto es, los mundos donde el grupo denotado es despreciado, ocupan un lugar más alto en el orden. Esto emparenta a los PG con proposiciones modales del tipo Los Ns deben ser despreciados o Los Ns son despreciables, donde $N$ refiere al grupo denotado por el PG, de modo análogo con otras emisiones con fuerza directiva (ver ejemplos

14 Como vimos en el caso de los imperativos, la actualización de la TDL no constituye necesariamente un compromiso a realizar una acción específica. Es razonable asumir que a menudo estas actitudes llevan a la acción, pero esto no es necesario. 
(8)-(11)). Esta relación parece constatarse por el hecho de que el uso de un PG licencia conversacionalmente tales modales:

30.

a. Juan es bolita.

b. Los bolivianos son despreciables.

31.

a. Mi oficina está llena de putos.

b. Los homosexuales deben ser despreciados ${ }^{15}$.

Finalmente, y dado que el compromiso asumido con este orden y con la proposición modal correspondiente es público, esta última es incorporada al $\mathrm{CG}$, alterando de este modo los hechos acerca de lo que es permisible al interior de la conversación.

Antes de proseguir, es importante realizar dos aclaraciones. Dijimos anteriormente que el uso de un PG de grupo actualiza tanto el CG como la TDL, siempre y cuando este sea comúnmente aceptado por el hablante y el destinatario. En primer lugar, la posición hasta aquí defendida no asume ninguna postura particular con respecto a las condiciones necesarias y suficientes, si las hubiere, para considerar que cierto uso de un PG fue de hecho aceptado (en lugar de rechazado u objetado) y, en consecuencia, que el contexto se actualizó de la manera esperada. Plausiblemente, esta es una cuestión contextual: en algunos contextos, la ausencia de un rechazo explícito cuenta como una aceptación; en otros, un silencio incómodo puede ser suficiente para hablar de un rechazo. En segundo lugar, la 'aceptación' a la que me refiero es conversacional, no moral. Aceptar el uso de un PG en este sentido acotado puede ser en algunos casos moralmente criticable, pero no necesariamente: en algunos contextos rechazar abiertamente el uso de un PG no es moralmente obligatorio, e.g. si el rechazo pone en riesgo la integridad física o la vida del individuo (para poner un ejemplo límite).

\subsection{Ventajas de la propuesta}

El enfoque aquí defendido presenta cierta analogía con la semántica y la pragmática de los imperativos. La propuesta es que los usos de PG tienen, por defecto, fuerza directiva: alientan ciertas actitudes, sentimientos o emociones 
y de este modo promueven el odio y la violencia. Esto es una consecuencia de la interacción entre su contenido semántico (la propiedad que expresan en su dimensión not at-issue) y los principios pragmáticos que regulan la dinámica misma de la conversación. En esta sección, argumentaré que la propuesta tiene varias ventajas.

En primer lugar, permite dar cuenta de la intuición según la cual los PG involucran un elemento modal en su interpretación. Esta idea es explícita en teorías como la de Hom y May (2013) y está implícita en otras, e.g. en la teoría de Langton, Haslanger y Anderson (2012), según la cual algunos usos de PG sirven para subordinar a sus destinatarios, o la teoría de Swanson (en prensa), según la cual los PG despliegan implicaturas de aceptabilidad (acceptability implicatures).

En segundo lugar, explica el modo en que los PG modifican a menudo hechos acerca de lo que es permisible. Los PG expresan una propiedad (la de despreciar al grupo relevante) de modo que, por defecto, tienen la función discursiva de actualizar la TDL. Así, estos usos, cuando son aceptados, se incorporan a la TDL correspondiente y comprometen públicamente a algunos de los participantes de la conversación a volver verdadera de sí mismos la propiedad de despreciar al grupo. La TDL así actualizada ordena entonces los mundos del CG de acuerdo con estos compromisos: los mejores mundos son aquellos donde el grupo afectado es despreciado. Sin embargo, la actualización de la TDL es un hecho manifiesto de la conversación e impacta por lo tanto en el $\mathrm{CG}$, que se actualiza incorporando una proposición modal acorde, del tipo Los Ns deberían ser despreciados, donde $\mathrm{N}$ denota al grupo afectado. De este modo, la propuesta explica cómo los PG incorporan contenidos modales en el CG, modificando así el conjunto de normas comúnmente aceptadas por los participantes de la conversación. Si esta legitimación local de normas discriminatorias en conversaciones específicas se difunde lo suficiente, puede tener un impacto significativo en la eventual emergencia de la norma a nivel global, o su legitimación.

En este punto, conviene detenernos para mencionar dos diferencias importantes entre mi posición y la defendida por Kirk-Giannini (2019). En primer lugar, de acuerdo con mi propuesta, qué TDL es actualizada por una emisión que contiene un PG es variable y depende del contexto. En algunos casos es la TDL del destinatario, o las TDL tanto del destinatario como del hablante, o ninguna. En la propuesta de Kirk-Giannini, en cambio, se actualizan las TDL de todos los participantes de la conversación. En segundo lugar, de acuerdo con mi enfoque, el tipo de modalidad involucrada en el acto directivo generado por el contenido peyorativo de los PG está abierto y puede variar 
de acuerdo con el contexto. En algunos casos puede ser deóntico, otras veces bulético, etc. Kirk-Giannini, en cambio, argumenta que el contenido directivo de los PG instruye a todos los participantes de la conversación a adoptar una perspectiva determinada acerca del grupo. En la línea de Camp (2013), Kirk-Giannini modela las perspectivas como modos de conceptualizar al grupo afectado. En su propuesta, las perspectivas determinan disposiciones a estructurar y organizar información, así como tendencias a juzgar ciertas emociones como apropiadas, entre otras cosas. En otras palabras, de acuerdo con Kirk-Giannini el contenido directivo instruye a todos los participantes de la conversación a conceptualizar al grupo afectado de cierto modo (de acuerdo con el hablante, el mejor modo de hacerlo).

En tercer lugar, la propuesta da cuenta de las diferentes fuerzas ilocucionarias asociadas con los PG. Estos generan al menos tres fuerzas ilocucionarias: propaganda, complicidad y ataque. Además, encontramos usos cómplices y apropiados. La fuerza ilocucionaria se deriva del contenido semántico, del contexto de emisión y de cuestiones pragmáticas relacionadas con las intenciones de los hablantes y principios generales de relevancia, racionalidad, cooperatividad, etc. Siguiendo a Davis y McCready (2017) y Poppa-Wyat y Wyatt (2017), asumiré que un factor fundamental en la determinación de la intención detrás del uso de un PG es la pertenencia (o no) del hablante/oyente al grupo denotado. Con esto en mente, consideremos las diferentes alternativas.

Una posibilidad es que ni el hablante ni el destinatario sean miembros del grupo subordinado. Esta situación encierra dos escenarios posibles: o bien el destinatario es manifiestamente prejuicioso o bien el hablante considera que el destinatario puede ser 'convencido' de adoptar ciertos prejuicios. En última instancia, el punto de este tipo de usos es crear o reforzar ciertas normas y/o disposiciones afectivas hacia el grupo subordinado. En ambos casos, la fuerza discursiva básica es la misma: el contenido peyorativo genera una fuerza discursiva directiva por defecto, esto es, actualiza la TDL de los participantes. Sin embargo, el contexto puede favorecer diferentes interpretaciones según la situación. La interpretación más natural del segundo caso (el hablante considera que el destinatario puede ser 'convencido') es la de una exhortación: el uso sirve para incitar al destinatario a adoptar una actitud peyorativa. En estos usos, la fuerza discursiva directiva de la emisión, sumada al conocimiento común de que el destinatario está abierto en principio a asumir compromisos discriminatorios, permiten derivar una interpretación propagandística del acto de habla.

En el primer caso (el destinatario es prejuicioso), la lectura más natural es la de la complicidad (Davis y McCready, 2017, pp. 4-5): la emisión funciona 
como una invitación a compartir un punto de vista prejuicioso. Un ejemplo de este tipo de usos es (32) utilizada por un hablante abiertamente xenófobo, y dirigida a un interlocutor con los mismos prejuicios:

32. Este país se llenó de bolitas.

Ahora bien, he argumentado que la fuerza discursiva por defecto de los PG es la de agregar una propiedad a la TDL de los participantes. En los casos de complicidad, sin embargo, la propiedad en cuestión (despreciar al grupo relevante) ya está presente en la TDL de ambos participantes. ¿Cuál es entonces el objetivo de estos usos? Hay dos posibilidades. En algunos casos, el objetivo de la emisión es agregar la información de que hablante y oyente comparten una posición discriminatoria con respecto al grupo al CG. Aun si ambos tienen una actitud discriminatoria y ambos creen que el otro tiene esta actitud, no necesariamente dicha información es CG. ${ }^{16}$ En estos casos, el punto de los usos cómplices es volver este hecho parte del CG haciendo explícitos los compromisos compartidos. La otra posibilidad es que las actitudes discriminatorias ya sean parte del CG. En estos casos el punto de los usos cómplices es mantener esa información en el CG. Plausiblemente, una proposición puede desaparecer del CG en diferentes etapas de la conversación, sea porque es rechazada explícitamente, sea porque deja de ser saliente o relevante. ${ }^{17}$ De este modo, repetir un PG no resulta redundante porque cumple la función de garantizar que las actitudes discriminatorias compartidas por los hablantes permanezcan en el CG. Ahora bien, en ambos casos (si el uso es aceptado) tanto la TDL del hablante como la del oyente son actualizadas con la propiedad relevante (despreciar al grupo en cuestión). Como se dijo anteriormente, esta actualización sirve una función conversacional, a saber, mantener en vigencia las actitudes discriminatorias comunes hacia el grupo denotado por el peyorativo, con las consecuencias normativas que esto conlleva.

Los usos como ataque son en principio problemáticos para la propuesta. Estos funcionan como meras expresiones de odio. Sin embargo, he defendido que los PG tienen por defecto una fuerza directiva, esto es, buscan típicamente lograr que otros adopten ciertas actitudes hacia el grupo subordinado.

16 Para que una proposición $p$ sea CG es necesario que todos crean que $p$, todos crean que todos creen que $p$, todos crean que todos creen que todos creen que $p$, y así sucesivamente, al infinito. Así, no es suficiente que los individuos en una conversación tengan una actitud discriminatoria y crean que el otro tiene dicha proposición para que esta sea CG entre ambos.

17 Como indica Schlenker (2012, p. 405), los hablantes son falibles, i.e. "En cualquier momento $t$ de una conversación, para cualquier proposición $p$ que era creída por el destinatario en $t-1$, existe una pequeña probabilidad de que este, por error, olvide $p . "$ 
¿Cómo explica este enfoque los usos como ataque? Argumentaré que estos casos funcionan de un modo análogo a ciertos usos de imperativos. Cuando un imperativo es emitido en un contexto en el cual es parte del CG que el destinatario no es capaz de llevar adelante las acciones requeridas o, en su defecto, que es muy poco probable que el destinatario tenga el deseo o la capacidad de realizarlas, aquel recibe a menudo una interpretación expresiva. Considere (33):

\section{3. ¡Disfrutá la película!}

Resulta difícil interpretar (33) con fuerza directiva, esto es, como una orden, una sugerencia, etc. En otras palabras, el hablante no parece buscar que el destinatario forme la intención de disfrutar la película, pues esto no es algo que dependa completamente de su voluntad. La interpretación más natural es, en cambio, que el hablante está expresando su deseo de que el destinatario disfrute la película. ${ }^{18}$ Esta reinterpretación resulta de la evaluación del contexto más principios conversacionales: dado que la interpretación default resulta inapropiada, el oyente buscará una interpretación alterativa que permita conservar el supuesto de que el hablante está siendo cooperativo. ${ }^{19}$

Algo similar ocurre, argumentaré, con los usos de PG como ataques. Un punto clave es que en estos casos el hablante no es un miembro del grupo discriminado mientras que el destinatario sí lo es, de modo que no existe una expectativa razonable de que este último forme la intención de discriminar a los miembros del grupo en cuestión (aunque la situación podría darse en casos muy raros). Más aun, el hecho antes mencionado es CG. En este contexto, la interpretación del PG con fuerza discursiva resulta implausible. En consecuencia, el destinatario buscará una interpretación alternativa que preserve el supuesto de cooperación, e.g. una interpretación expresiva. Por último, dado que el hablante está empleando una expresión que codifica un contenido peyorativo, es razonable suponer que la emisión será interpretada como un ataque. Así, a diferencia de los usos de PG con fuerza directiva, en este caso el hablante no busca que el destinatario adopte normas o actitudes

18 La posibilidad de reinterpretar la fuerza discursiva de una oración es un fenómeno general plenamente establecido. Así, una oración interrogativa '¿Me podés pasar la sal?' puede reinterpretarse como un pedido en lugar de una pregunta, y una oración declarativa como 'Te vas a sentar y vas a hacer la tarea' puede reinterpretarse como una orden en ciertos contextos (e.g. dicho por un padre a su hijo). En el caso de (33), la emisión es reinterpretada, para ponerlo en términos intuitivos, como 'Ojalá disfrutes la película'.

19 Cabe realizar la siguiente aclaración: sin dudas, insultar a otro individuo no es una actividad cooperativa. Cuando digo a que el intercambio es cooperativo, me refiero a que el hablante está apoyándose en el reconocimiento mutuo de intenciones comunicativas para transmitir un contenido a través de un acto de habla que resulte pragmáticamente apropiada en el contexto. 
discriminatorias hacia el grupo, sino que simplemente busca manifestar sus propias actitudes discriminatorias, con el fin de ofender al destinatario. Finalmente, dado que el acto de habla de ataque es un hecho manifiesto, este impacta en el $\mathrm{CG}$, que se actualiza con algunas proposiciones implicadas pragmáticamente, e.g. que el hablante desprecia al grupo en cuestión.

Finalmente, la variabilidad con respecto a la pertenencia del hablante y/o el oyente al grupo discriminado permite explicar los usos apropiados. En estos casos, al igual que en los usos como ataque, la fuerza directiva es reinterpretada contextualmente. Por un lado, la pertenencia del destinatario al grupo subordinado bloquea la expectativa de que este agregue la propiedad relevante a su TDL. De acuerdo con lo anterior, esto habilita una interpretación expresiva. Sin embargo, la interpretación como ataque también es cancelada en estos casos: siendo el hablante también un miembro del grupo discriminado, es improbable que su emisión sea interpretada como un insulto. Estos hechos, junto con el conocimiento contextual de la historia social del PG usado, habilitan una interpretación de la emisión como una expresión de solidaridad o camaradería entre miembros de un grupo oprimido.

En este punto, es nuevamente necesario un breve comentario sobre el trabajo de Kirk-Giannini. El autor motiva su posición argumentando en contra del expresivismo orientado al hablante (cf. Jeshion, 2013), i.e., el enfoque según el cual la función primaria de los PG es la de expresar un estado mental negativo sobre el hablante (sea una creencia u otro tipo de actitud). En pocas palabras, Kirk-Giannini mantiene que el expresivismo tiene dos problemas: primero, no explica por qué una emisión que expresa la misma información que otra que contiene un PG carece del potencial discriminatorio de esta última; segundo, no explica por qué los usos de PG son ofensivos aun en contextos en los cuales las actitudes del hablante son conocidas de antemano. Mi propuesta da cuenta de estas dos cuestiones. Por un lado, argumenté que los PG tienen una fuerza discursiva directiva, de manera que estos términos no tienen solamente la función primaria de expresar las actitudes del hablante. Por el otro lado, defendí que en algunos casos (los usos como ataque y los usos apropiados) los PG cumplen la función de expresar una actitud del hablante. Sin embargo, si la presente propuesta es correcta, el modo en que ocurre esto último evita los problemas que señala Kirk-Giannini para el expresivismo orientado al hablante. Más precisamente, los PG cumplen una función expresiva solamente en algunos contextos y el contenido en cuestión es expresado de manera pragmática, y no codificado en el significado convencional. Por otra parte, cabe destacar que algunos usos representan un desafío para la posición de Kirk-Giannini: en su propuesta, los usos de PG tienen siempre una fuerza ilocucionaria directiva 
(promueven la adopción de cierta perspectiva cognitiva hacia el grupo discriminado). Sin embargo, parece haber algunos usos que funcionan más bien como puros insultos, expresiones ciegas de odio y violencia.

\section{Roles discursivos y discurso de odio}

En esta última sección discutiré brevemente la posición de Poppa-Wyatt y Wyatt (2017). Los autores buscan dar cuenta de varios fenómenos relacionados con el uso de PG a través de la noción de rol discursivo. Un rol discursivo asigna cierto estatus o jerarquía a un individuo o grupo en relación con otros. En el caso de los PG, el rol discursivo asignado caracteriza a cierto grupo como subordinado/dominante en relación con otros. Además, los roles discursivos determinan parcialmente las reglas que gobiernan la conversación: las movidas conversacionales esperadas, las movidas conversacionales permitidas, las reglas de interpretación relevantes, los efectos esperados de las diversas contribuciones conversacionales, etc. Así, por ejemplo, la oración 'Quiero 1000 dólares', será interpretada de un modo diferente de acuerdo a los roles sociales de los interlocutores, e.g. si el diálogo se da entre un cajero de banco y un cliente, o entre el primero y un ladrón (Popa-Wyatt y Wyatt, 2017, p. 11).

Los roles discursivos son internos a la conversación y de corta duración, a diferencia de los roles sociales externos, de larga duración. Los primeros se crean cuando cierto rol social externo se hace saliente en la conversación, quedando asociado a alguno de los participantes. Así, los roles discursivos dependen en algún sentido de los roles sociales. Sin embargo, se da una dinámica de retroalimentación donde la asignación de roles discursivos de corta duración depende en parte de la existencia de roles sociales externos previos, y a su vez contribuye a la legitimación de estos últimos.

La tesis principal de Poppa-Wyatt y Wyatt es que los usos de PG buscan crear o mantener una distribución de poder injusta, a través de la asignación de roles discursivos a los participantes de la conversación. La idea es que los PG instancian una fuerza ilocucionaria ejercitativa (excercitive), que asigna un rol al destinatario y, complementariamente, al hablante. Así, una oración como (34):

34. ¡Calláte, maldito maricón!

(Popa-Wyatt y Wyatt, 2017, p. 15) confiere un rol subordinado al destinatario y un rol dominante al hablante, a la vez que impacta en la determinación de las movidas conversacionales permisibles para uno y otro, así como en las reglas de interpretación de sus 
emisiones y los efectos esperados de estas. De este modo, el uso de un PG implica una modificación en la distribución de poder entre los participantes de la conversación que impacta en los hechos acerca de qué movimientos son permisibles de allí en más. Por este motivo, Poppa-Wyatt y Wyatt sostienen que la asignación de un rol discursivo es una movida conversacional de segundo orden (meta-move), que modifica el score conversacional, en términos de Lewis (1979), pero modifica además las reglas específicas que gobiernan la conversación.

La posición permite, en principio, explicar cómo es que el uso de PG constituye un tipo de discurso opresivo: al asignar roles discursivos subordinados a ciertos grupos, refuerza una distribución injusta de poder y retroalimenta la asignación de roles sociales externos subordinados al grupo discriminado, roles que prescriben daños materiales, políticos, psicológicos, epistémicos, etc., a este. De acuerdo con los autores, la teoría permite además explicar diferentes tipos de variabilidad en la ofensa impartida por los PG: PG para diferentes grupos varían en el grado de ofensa generado, como también lo hacen diferentes peyorativos para el mismo grupo. Además, diferentes usos del mismo PG por diferentes hablantes, o dirigidos hacia diferentes audiencias, generan ofensas de distinta intensidad. La teoría mantiene que la variabilidad en la ofensa corresponde al nivel de injusticia percibida en la distribución de poder que el uso del peyorativo busca imponer. Diferentes peyorativos, sea para el mismo grupo o para grupos diferentes, están asociados con diferentes roles y promueven así diferentes formas de distribución de poder. Este criterio general, con algunos matices, permite también explicar, según a los autores, fenómenos como los usos apropiados y el fenómeno del silenciamiento.

La teoría tiene varias ventajas explicativas. Argumentaré, sin embargo, que presenta dos problemas fundamentales. Primero, es posible generar oraciones que asignan roles discursivos subordinados sin usar PG. Estas oraciones, sin embargo, no presentan el comportamiento lingüístico o el efecto conversacional de las oraciones que sí involucran PG. La propuesta de Poppa-Wyatt y Wyatt no puede dar cuenta de esta diferencia. Segundo, hay usos de PG que no asignan los roles relevantes - por ejemplo, algunos contenidos negados o atribuciones de creencia - pero que, sin embargo, son ofensivos. Si la conducta lingüística y la ofensa estuvieran determinadas por la capacidad de los PG para asignar roles discursivos, no deberían observarse estas diferencias. En términos más generales, el problema de la teoría es que no proporciona una semántica para los PG, de modo que toda la conducta de estos debe derivarse a partir de los roles discursivos y otros factores contextuales mencionados por los autores. Pero esto, por sí solo, no permite distinguir a los PG de otros casos de discurso 
opresivo que prescinden de PG. Estos problemas sugieren, en línea con la teoría defendida en las secciones anteriores, que es necesario apelar al significado convencional de los PG para dar cuenta de su conducta de manera acabada. Veamos ambas críticas con más detalle.

En primer lugar, es posible asignar roles discursivos sin necesidad de usar PG. Considere (35):

35. Los bolivianos deben ocupar un rol subordinado al resto de los ciudadanos.

Sin dudas, (35) busca asignar un rol subordinado a las personas nacidas en Bolivia. Sin embargo, está claro que no se comporta del mismo modo que una oración como (23). Por ejemplo, es posible negar (35) de manera directa, sin alterar el flujo normal de la conversación:

36. No es cierto, los bolivianos no deben ocupar un rol subordinado al resto de los ciudadanos.

Del mismo modo, los PG están fuertemente atados al hablante, mientras que una oración como (35) no lo está. Esto puede verse, por ejemplo, al considerar su comportamiento bajo verbos de creencia:

37. Juan cree que los bolitas deben ocupar un lugar subordinado al resto de los ciudadanos.

38. Juan cree que los bolivianos deben ocupar un lugar subordinado al resto de los ciudadanos.

(37) expresa una actitud discriminatoria del hablante, y no solamente del sujeto de la adscripción, mientras que (38) solo permite adscribir una actitud discriminatoria a este último.

Por otra parte, aun cuando (35) resulta ofensiva, parece mostrar un nivel de ofensa menor que una oración como (39):

39. Los bolitas deben ocupar un rol subordinado al resto de los ciudadanos. El problema general es el siguiente. La teoría de Poppa-Wyatt y Wyatt mantiene que las emisiones con PG son ofensivas porque asignan roles subordinados a ciertos grupos. Si esto fuera cierto, otras oraciones que asignan roles subordinados deberían ser ofensivas en el mismo grado. Esto, sin embargo, no ocurre. Al mismo tiempo, las oraciones con PG presentan un comportamiento lingüístico específico, diferente de otras oraciones que permiten asignar roles 
discursivos. Al no proporcionar una semántica explícita para los PG, la teoría es incapaz de dar cuenta de estas diferencias.

El segundo problema es que existen usos de PG que no asignan un rol discursivo al destinatario $\mathrm{y}$, sin embargo, son ofensivos:

40. Los bolitas no deben ocupar un rol subordinado. De hecho, ningún grupo debe ocupar un lugar subordinado puramente en virtud de su nacionalidad.

Considérese un uso sincero de (40) en el que, además, son perfectamente claras las intenciones comunicativas del hablante. En tal contexto, (40) no asigna un rol subordinado al destinatario o al grupo. En particular, una emisión como (40) no parece hacer permisible en la conversación ulterior discriminar de ningún modo a las personas de nacionalidad boliviana. Aun así, la emisión resulta ofensiva. Ciertamente, la continuación de la oración inicial matiza bastante el efecto ofensivo. Aun así, el uso de 'bolita' puede ser percibido como inapropiado. Uno podría imaginar una respuesta del siguiente tipo: 'Coincido con vos, pero no deberías referirte a los bolivianos como 'bolitas', es ofensivo.'

Otro ejemplo, tal vez un poco más controvertido, puede verse en los casos de mención. En el contexto cultural norteamericano el PG 'Nigger' no puede siquiera ser mencionado por personas blancas sin generar ofensa. Sin embargo, está claro que la mera mención no asigna un rol subordinado al grupo: plausiblemente, para que un PG instancie una fuerza ilocucionaria ejercitativa del tipo requerido la expresión debe ser $u s a d a .^{20}$

La teoría de Poppa-Wyatt y Wyatt reconoce un rol importante a diferentes elementos relacionados con la dinámica conversacional en la explicación del modo en que funcionan los PG. Sin embargo, la explicación falla como propuesta general para dar cuenta de su funcionamiento. La falla se debe, de acuerdo a lo argumentado más arriba, a la carencia de una semántica explícita para los PG. Al incorporar una propuesta acerca del significado convencional de los PG y otorgarle un papel relevante en la teoría, la posición defendida en las secciones anteriores permite dar cuenta de fenómenos conversacionales como los que interesan a los autores y, a la vez, dar cuenta del comportamiento lingüístico de estas expresiones, diferente de otras instancias de discurso de odio.

20 Como señala un referí anónimo, cabe aclarar que este tipo de ejemplos depende fuertemente del contexto social. En este caso, el fenómeno es relativo al código cultural norteamericano. En dicha sociedad algunas palabras funcionan como tabú, de modo que no pueden siquiera ser mencionadas sin generar ofensa. Esto cambia radicalmente en el contexto hispanoamericano, donde es imposible encontrar este tipo de prohibiciones. 


\section{Conclusión}

En este artículo argumenté que los PG tienen dos dimensiones de significado, una dimensión que codifica una propiedad descriptiva que denota a un grupo y una dimensión peyorativa que codifica la propiedad de despreciar al grupo en cuestión. En el marco de una teoría pragmática dinámica, se sigue que las oraciones que contienen PG instancian simultáneamente dos funciones discursivas, esto es, actualizan el contexto de dos maneras diferentes. Por un lado, actualizan el CG; por el otro, actualizan la TDL. Esto significa que los PG poseen en un nivel una fuerza discursiva directiva por defecto, de manera revocable. De este modo, estas expresiones resultan especialmente adecuadas para incitar a los destinatarios a adoptar ciertas actitudes.

La propuesta tiene varias ventajas. Primero, proporciona una explicación pragmática del modo en que los PG modifican hechos acerca de lo que es permisible (a través de su impacto modal en el CG) sin descuidar el papel que cumple su contenido convencional en el proceso. Segundo, explica el perfil ilocucionario de esas expresiones: la propaganda y los usos cómplices explotan la fuerza directiva con diferentes propósitos, para exhortar al hablante a despreciar al grupo en un caso, para volver el compromiso discriminatorio parte del CG (o conservarlo allí) en el otro. La posición también arroja luz sobre los usos como ataque y los usos apropiados. En estos casos, la fuerza discursiva directiva es reinterpretada debido a factores contextuales relacionados con la pertenencia (o no) del hablante o el destinatario al grupo discriminado, arrojando así una lectura expresiva de diferente tipo en cada caso, de manera análoga a ciertos usos de imperativos.

Finalizaré el artículo discutiendo brevemente la distinción entre discursos que causan daño y discursos que constituyen un daño en sí mismos. Como fue señalado en la introducción, un discurso constituye un daño en sí mismo en la medida en que crea o refuerza, en virtud de su fuerza ilocucionaria, normas sociales que prescriben un daño, mientras que un discurso meramente causa daño cuando este es un efecto perlocucionario del acto de habla. La propuesta es compatible con esta distinción. De acuerdo con lo argumentado más arriba, varios usos de PG (e.g. los propagandísticos) instancian una fuerza discursiva directiva. En virtud de esto, modifican a veces hechos acerca de lo que es permisible en virtud de su contenido semántico y el modo en que actualizan el contexto. En otras palabras, el uso de PG tiene un impacto normativo en la conversación. Aun cuando el efecto inmediato de este proceso es local, esto es, se circunscribe a la conversación, se retroalimenta con normas sociales externas y de largo plazo. Así, al menos algunos usos de PG instituyen o legitiman 
normas sociales que prescriben un daño para individuos y grupos en virtud de su fuerza discursiva. En otras palabras, constituyen un daño.

Los usos como ataque son diferentes. En esos casos, la fuerza directiva default es reinterpretada por factores contextuales, de modo que el acto de habla acaba por cumplir una función discursiva expresiva. Estos usos son sin dudas dañinos, pero de un modo diferente a los usos propagandísticos. Plausiblemente, la expresión de odio incita a otros al odio y la violencia. Por ejemplo, si el uso proviene de un individuo que goza de respeto y autoridad en la comunidad puede llevar a otros a imitarlo. Sin embargo, este mecanismo de diseminación del odio no depende de las características lingüísticas del acto de habla, sino que puede ser considerado como un efecto perlocucionario de la emisión. Si esto es correcto, los usos como ataque no constituyen un daño, sino que meramente lo causan.

\section{Bibliografía}

ANAND, P. “Re-expressing Judgment.” Theoretical Linguistics, 33(2), 2007, pp. 199-208.

ANDERSON, L. and LEPORE, E. "Slurring Words." Noûs, 47(1), 2013, pp. 25-48.

BIANCHI, C. "Slurs and appropriation: An echoic account." Journal of Pragmatics, 66, 2014, pp. 35-44.

BOLINGER, R. J. “The Pragmatics of Slurs”. Noûs, 51(3), 2017, pp. 439-462.

BRONTSEMA, R. "A queer revolution: Reconceptualizing the debate over linguistic reclamation." Colorado Research in Linguistics 17, 2004. DOI: https://doi.org/10.25810/ dky3-zq57

CAMP, E. "Slurring Perspectives," Analytic Philosophy, 54(3), 2013, pp. 330-349.

CASO, R. "A Bidimensional Accounts of Surs.” In: E. Orlando y A. Saab (eds.), en prensa.

CELIKATES, R. (ed.). “A Volume on Ideology”. Oxford: Oxford University Press, en prensa.

CEPOLLARO, B. "In Defense of a Presuppositional Account of Slurs." Language Sciences, 52, 2015, pp. 36-45.

. "The Semantics and Pragmatics of Slurs and Thick Terms", 2017. PhD dissertation. https://tel.archives-ouvertes.fr/tel-01508856/document.

CEPOLLARO, B. y THOMMEN, T. "What's Wrong with Truth-Conditional Accounts of Slurs?" Linguistics and Philosophy, 42(4), 2019, pp. 333-347.

DAVIS, C. y MCCREADY, E. Proceedings of LENLS14, 2017.

GRAFF FARA, D. y RUSSELL, G. (eds.). "Routledge Companion to Philosophy of Language". Dordrecht: Routledge, 2012.

GUTZMANN, D. Use-Conditional Meaning. Oxford: Oxford University Press, 2015. HARRIS, D. FOGAL, D. y MOSS, M. (eds.). "New Work on Speech Acts". Oxford: Oxford University Press, 2018. 
HOM, C. "The Semantics of Racial Epithets." Journal of Philosophy, 105(8), 2008, pp. 416-440.

HOM, C. y MAY, R. "Moral and Semantic Innocence." Analytic Philosophy, 54, 2013, pp. 293-313.

JESHION, R. "Expressivism and the Offensiveness of Slurs." Philosophical Perspectives, 27, 2013, pp. 232-259

KIRK-GIANNINI, C. D. “Slurs are Directives.” Philosophers'Imprint, 19(48), 2019, $1-28$.

KRATZER, A. "Beyond 'Ouch' and 'Oops': How Descriptive and Expressive Meaning Interact." Handout for Cornell Conference on Theories of Context Dependency, Amherst, 1999.

. Modals and Conditionals. Oxford: Oxford University Press, 2012.

KUKLA, R. "Slurs, Interpellation, and Ideology." The Southern Journal of Philosophy, 56, 2018, pp. 7-32.

LANGTON, R. "How to Get a Norm from a Speech Act." The Amherst Lecture in Philosophy, 10, 2015, pp. 1-33. . "Speech Acts and Unspeakable Acts." Philosophy \& Public Affairs, 22(4), 1993, pp. 293-330.

LANGTON, R. y WEST, C. "Scorekeeping in a Pornographic Language game." Australasian Journal of Philosophy, 77(3), 1999, pp. 303-319.

LANGTON, R., HASLANGER, S. y ANDERSON, L. "Language and Race.” In: G. Rusell y D. Graff Fara (eds.), 2012, pp. 753-767.

LEWISS, D. K. "Scorekeeping in a language game." Journal of Philosophical Logic, 8(1), 1979, pp. 339-359.

LO GUERCIO, N. "Slurs and antipresuppositions." Natural Language Semantics, 29, pp. 377-400, 2021. https://doi.org/10.1007/s11050-021-09178-y

MACKINNON, C. A. "Feminism Unmodified: Discourses on Life and Law". Cambridge, Mass.: Harvard University Press, 1987.

MAITRA, I. "Subordinating Speech.” In: I. Maitra y M. K. McGowan (eds.), 2012, pp. 94-120.

MAITRA, I. y MCGOWAN, M. K. (eds.). "Speech and Harm: Controversies over Free Speech". Oxford: Oxford University Press, 2012.

MATSUDA, M. "Public Response to Racist Speech: Considering the Victim's Story." En: M. Matsuda (ed.), 2018, pp. 17-51.

. "Words that Wound: Critical Race Theory, Assaultive Speech, and the First Amendment", Boulder: Westview Press, 2018.

MCCREADY, E. "Varieties of Conventional Implicature." Semantics and Pragmatics, 3, 2010, pp. 1-57.

MCGOWAN, M. K. "Conversational Exercitives: Something Else We Do with Our Words." Linguistics and Philosophy, 27(1), 2004, 93-111.

. "Just Words: on Speech and Hidden Harm". Oxford: Oxford University Press,

2019. 
NUNBERG, G. "The Social Life of Slurs.” In: D. Harris, D. Fogal y M. Moss (eds.), 2018, pp. 237-295.

ORLANDO, E. y SAAB, A. (eds.), "Slurs and Expressivity: Semantics and Beyond". Lexington, en prensa.

ORLANDO, E. y SAAB, A. "Slurs, Stereotypes and Insults." Acta Analytica, 2020. https://doi.org/10.1007/s12136-020-00424-2.

POPPA-WYATT, M. y WYATT, J. L. "Slurs, roles and power." Philosophical Studies, 175(11), 2018. https://doi.org/10.1007/s11098-017-0986-2.

PORTNER, P. “Imperatives and Modals.” Natural Language Semantics, 15(4), 2007, pp. 351-383.

. "Mood". Oxford University Press, 2018.

"The Semantics of Imperatives within a Theory of Clause Types." En: K.

Watanabe y R. B. Young (eds.), 2004, pp. 235-252.

POTTS, C. "The Logic of Conventional Implicatures". Oxford: Oxford University Press, 2005.

PREDELLI, S. "Meaning Without Truth”. Oxford: Oxford University Press, 2013.

ROEBRTS, C. "Speech Acts in Discourse Context." In: D. Harris, D. Fogal y M. Moss (eds.), 2018, pp. 317-259.

SAAB, A. "On the Locus of Expressivity. Deriving Parallel Meaning Dimensions from Architectural Considerations." En: E. Orlando y A. Saab (eds.), en prensa.

SCHLENKER, P. “Expressive Presuppositions.” Theoretical Linguistics, 33(2), 2007, pp. 237-245.

. "Maximize Presupposition and Gricean Reasoning." Natural Language

Semantics, 20, 2012, pp. 391-429.

SENNET, A. y COPP, D. "What Kind of a Mistake is It to Use a Slur?" Philosophical Studies, 172(4), 2015, pp. 1079-1104.

STALNAKER, R. C. "Context and Content: Essays on Intentionality in Speech and Thought". Oxford: Oxford University Press, 1999.

SWANSON, E. "Slurs and Ideologies." En: R. Celikates (ed.), en prensa.

TIRRELL, L. "Genocidal Language Games." En: I. Maitra y M. K. McGowan (eds.), 2012, pp. 174-221.

VON FINTEL, K. "What is Presupposition Accommodation, Again?" Philosophical Perspectives, 22(1), 2008, pp. 137-170.

WATANABE, K. y YOUNG, R. B. (eds.), "Proceedings of Semantics and Linguistic Theory 14", CLC Publications, 2004. 